\title{
Editorial
}

\section{Morrey Spaces and Related Function Spaces}

\author{
Yoshihiro Sawano, ${ }^{1}$ Hendra Gunawan, ${ }^{2}$ Vagif Guliyev, ${ }^{3,4}$ and Hitoshi Tanaka ${ }^{5}$ \\ ${ }^{1}$ Department of Mathematics and Information Scinence, Tokyo Metropolitan University, 1-1 Minami-Ohsawa, Hachioji 192-0397, Japan \\ ${ }^{2}$ Department of Mathematics, Bandung Institute of Technology, Bandung 40132, Indonesia \\ ${ }^{3}$ Department of Mathematics, Ahi Evran University, Kirsehir, Turkey \\ ${ }^{4}$ Institute of Mathematics and Mechanics of NAS of Azerbaijan, Baku, Azerbaijan \\ ${ }^{5}$ Graduate School of Mathematical Sciences, The University of Tokyo, Tokyo 153-8914, Japan
}

Correspondence should be addressed to Yoshihiro Sawano; yoshihiro-sawano@celery.ocn.ne.jp

Received 29 January 2014; Accepted 29 January 2014; Published 19 May 2014

Copyright (C) 2014 Yoshihiro Sawano et al. This is an open access article distributed under the Creative Commons Attribution License, which permits unrestricted use, distribution, and reproduction in any medium, provided the original work is properly cited.

\section{Introduction}

In connection with elliptic partial differential equations, C. Morrey proposed a weak condition for the solution to be continuous enough in 1938. Later on, his condition became a family of normed spaces and they are called Morrey spaces. Although the notion is originally from the partial differential equations, the space turned out to be important in many branches of mathematics. In fact, in these five years more than 500 papers are written about Morrey spaces. The aim of this special issue is to take up the recent development of Morrey spaces.

From the recent papers, we feel that the following topics are remarkable.

(1) Equivalent expression of Morrey spaces.

(2) Precise estimate of the Riesz potential operators.

(3) Morrey spaces as initial value.

(4) Multilinear operators.

(5) Weighted estimates.

(6) Morrey spaces on general geometric measure spaces.

(7) Role of each parameter.

(8) Related function spaces.

Although some of them overlap, we describe them in more details.

\section{Details of the Development}

2.1. Equivalent Expression of Morrey Spaces. Starting from the equivalent expression by means of Littlewood-Paley characterizations according to A. Mazzucato, many theories appeared in connection with the characterization by way of the Carleson type characterization with BMO spaces. One of the prominent achievements is the definition of BesovMorrey spaces according to H. Kozono and M. Yamazaki and the definition of Triebel-Lizorkin-Morrey spaces according to L. Tang and J. Xu. Later Triebel-Lizorkin-Morrey spaces emerged to Triebel-Lizorkin type spaces proposed by D. Yang and W. Yuan. The theory of Triebel-Lizorokin-Morrey spaces and Besov-Morrey spaces are expanded by T. Ullrich, Y. Liang, D. Haroske, Y. Sawano, W. Sickel and L. Skrzypczak. Especially, the embedding relation is intensively investigated.

2.2. Precise Estimate of the Riesz Potential Operators. In 1975 , D. Adams proved the Morrey boundedness of the Riesz potential operators, which was the motivation of working on Morrey spaces. This result extends the result by Spanne. The result by Spanne is recorded by Peetre. Although it is very fundamental in partial differential equations to express the solution of the Laplace equations by way of Riesz potentials, we are faceing the situation where the classical results are not available or not enough. In 1994 E. Nakai proposed the generalized Morrey spaces and in 2002. E. Nakai also proposed the generalized fractional integral operators. V. 
Guliyev proposed the weak type variant in parallel in 1994. Y. Sawano and H. Wadade by some examples in partial differential equations showed that these notions are not mere quests to generality.

After E. Nakai proposed the definition, H. Gunawan proposed a sufficient condition for a bilinear operator generated by a fractional integral operator to be bounded. Later, Eridani, H. Gunawan, E. Nakai, and Y. Sawano showed that the condition is necessary as well.

2.3. Morrey Spaces as Initial Value. No matter how we choose the parameter $p \in(0, \infty]$, the function $|x|^{-a}$ does not belong to $L^{p}\left(\mathbb{R}^{n}\right)$. However, Morrey spaces can contain such functions. Therefore, some authors use Morrey spaces to describe the initial condition of partial differential equations.

2.4. Multilinear Operators. More and more attention is paid to multilinear operators after the solution of the Carleson conjecture on the boundedness of bilinear Hilbert transform. In this connection, many people started to investigate the multilinear singular integral operators, the multilinear fractional integral operators, and so on. One of the advantages to work on Morrey spaces with fractional integral operators is that the less additional assumption is necessary when the multiplicity is greater than 2 .

Another crucial progress is a bilinear estimate initiated by P. Olsen, which is later sharpened by H. Tanaka in 2009. When we try to ensure the existence of partial differential equations, we sometimes need to handle the pointwise product. In such a case, we need a weaker integrability of one of two functions. It seems that Morrey spaces are suitable to describe the weaker integrability.

2.5. Morrey Spaces on General Geometric Measure Spaces. Morrey spaces are equipped with two parameters. One of them seems to describe the local regularity and therefore Morrey spaces can be used to describe the integrability more precisely than Lebesgue spaces. This fact is illustrated by many examples including ones from partial differential equations. When we work on general geometric measure spaces $(X, d, \mu)$, it is not the case that the measure $\mu$ is not doubling; $\mu(B(x, 2 r)) \leq C \mu(B(x, r))$ fails. When we place ourselves on the Euclidean space, Y. Sawano and $H$. Tanaka proposed a solution to overcome this difficulty. Later many people culminated the results and nowadays these spaces are called Morrey spaces with nondoubling measures. See the works of Shimomura in connection with fractional integral operators and non-doubling measures. Sawano and Shimomura gave an example that some of fundamental results do not hold in the generalized setting.

2.6. Role of Each Parameter. It is very important to consider the role of two parameters which Morrey spaces have. The spaces $\mathscr{M}_{1}^{p}$ and $\mathscr{M}_{2}^{p}$ are of extreme importance.

2.7. Weighted Estimates. Among the geometric measure spaces, the weighted Morrey spaces are of interest. Y. Komori and S. Shirai proposed a sufficient condition in 2009, under which many operators are later shown to be bounded by many people. In particular, the characterization of the boundedness of maximal operators is very important.

2.8. Related Function Spaces. To have a better understanding, many other related function spaces are proposed. For example, K. Matsuoka and E. Nakai defined $B_{\sigma}$-spaces. V. Guliyev and V. Burenkov considered local Morrey spaces. The predual of Morrey spaces is proposed by C. Zorko. E. Kalita in 1998 and D. Adams and J. Xiao in 2004 obtained equivalent expressions. In 2012 R. Mustafayev and A. Gogatishvili investigated the predual more in detail. In 2013 T. Izumi, E. Sato, and K. Yabuta proved that the predual space is closed under the monotone limit.
Yoshihiro Sawano Hendra Gunawan Vagif Guliyev Hitoshi Tanaka 


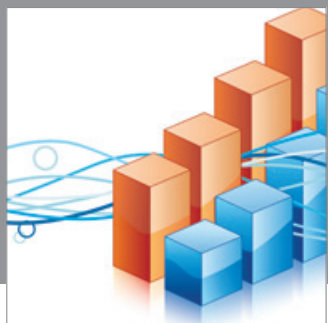

Advances in

Operations Research

mansans

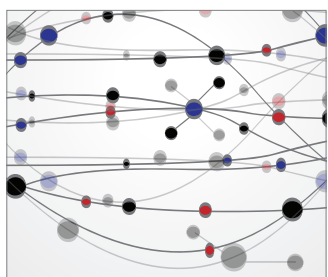

The Scientific World Journal
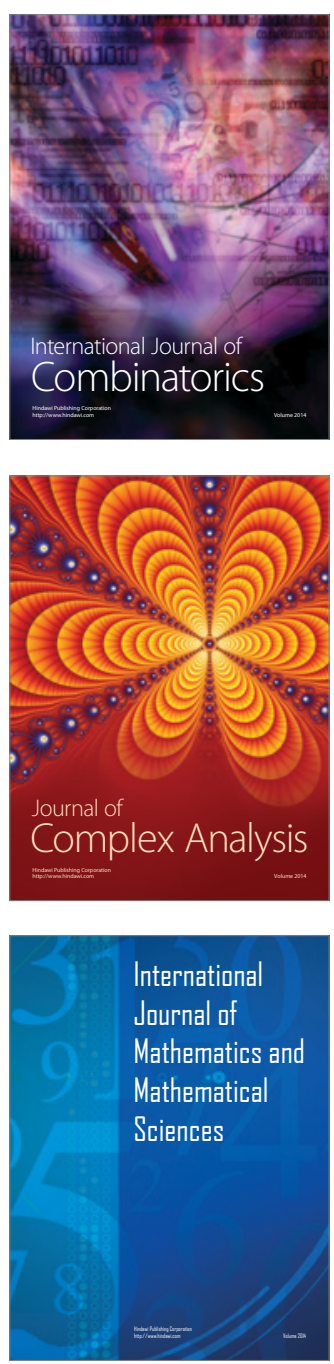
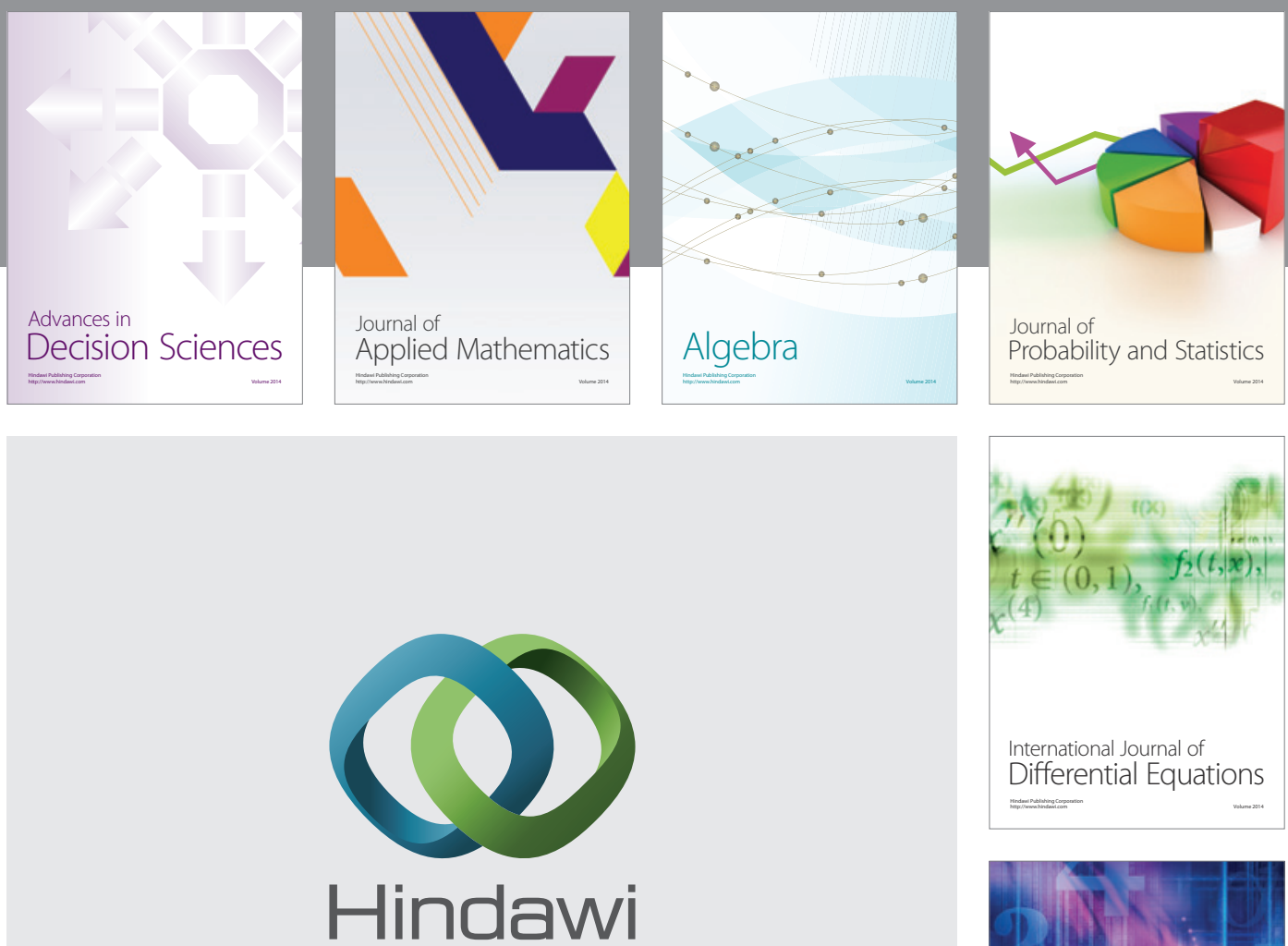

Submit your manuscripts at http://www.hindawi.com
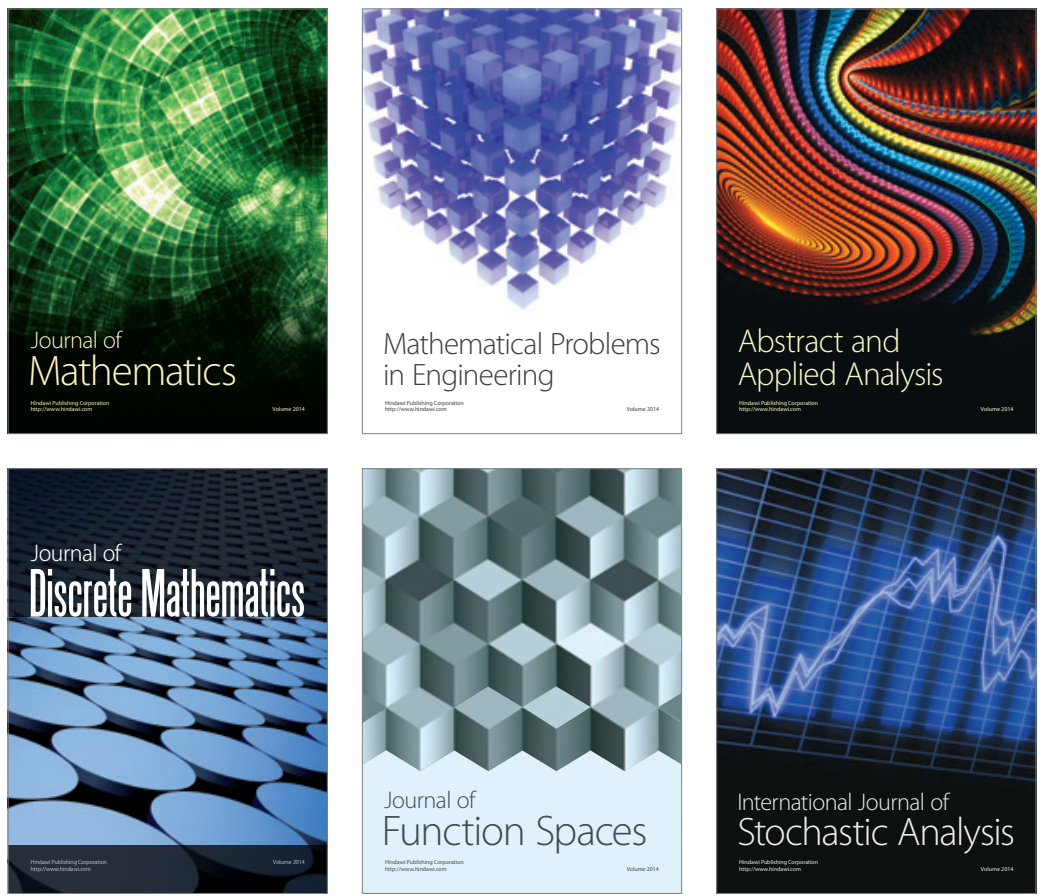

Journal of

Function Spaces

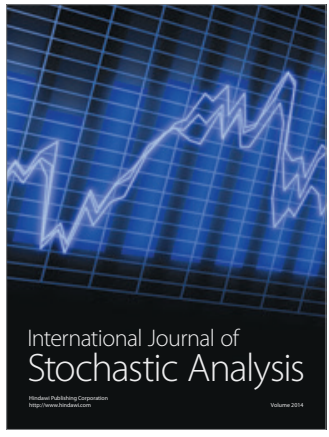

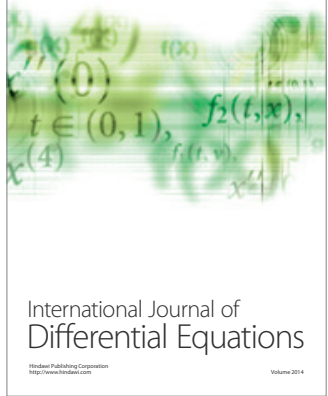
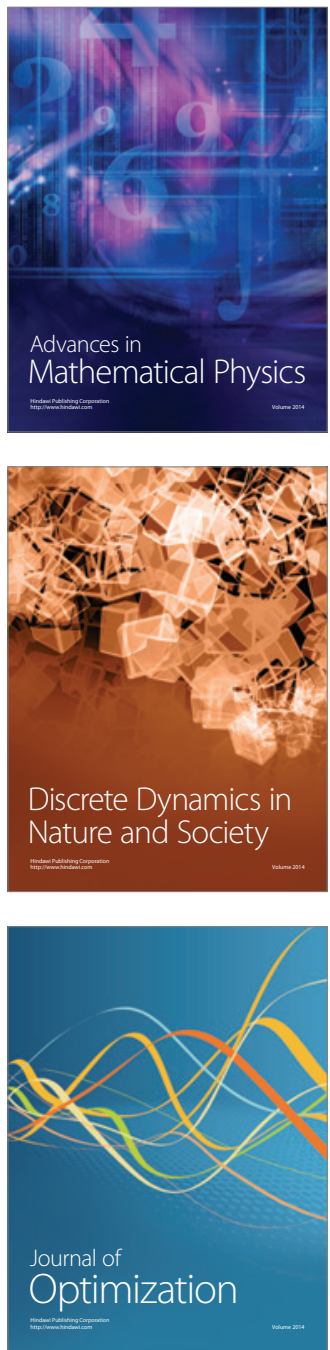